\title{
La Ansiedad en la Interpretación Musical: programa de intervención en un coro
}

\author{
Laura Fernández-Granados ${ }^{1}$; Carolina Bonastre ${ }^{2}$
}

Recibido: 29/3/2020 / Aceptado: 8/7/2020

Resumen. La Ansiedad en la Interpretación Musical (AIM) consiste en la aprensión ansiosa vinculada a las expresiones musicales padecida por músicos y cantantes, afectando a sus niveles de bienestar, concentración y calidad interpretativa. El presente estudio consiste en el diseño e implementación de una intervención sobre la AIM de un grupo de coristas aficionados, estudiantes y profesionales. Se empleó método mixto, mediante instrumentos de recogida de datos antes y después del programa. Los resultados señalan que: (a) los comentarios de la evaluación previa reflejan preocupación por la exposición en público y síntomas de AIM; (b) tras la intervención se redujeron significativamente las puntuaciones de ítems de las escalas Vulnerabilidad Psicológica, Pensamientos Específicos y Motor y Fisiológico; (c) se logra una adquisición de técnicas de afrontamiento adaptativas; y (d) el grupo de discusión valora positivamente la experiencia. El programa analizado se propone como una estrategia sencilla y breve para ayudar al control de la AIM.

Palabras clave: Educación musical; Psicología de la educación; Intervención; Ansiedad; Música vocal; Ambiente profesional.

\section{[en] Music Performance Anxiety: an intervention program in a choir}

Abstract. Music Performance Anxiety (MPA) consists of anxious apprehension linked to musical expressions suffered by musicians and singers, affecting their levels of well-being, concentration and interpretive quality. This study focuses on the design and implementation of an intervention based on the MPA of amateur choristers, music students and professionals. Mixed methods were used through several instruments before and after the program to gather information. The results indicate that: (a) items of the Psychological Vulnerability, Specific Cognitions and Motor and Physiological scales showed significant differences in their scores after the intervention; (b) comments from the previous evaluation reflect concern about performing in public and symptoms of MPA; (c) an acquisition of adaptive coping techniques and methods is achieved; and (d) the discussion group values the experience positively. The analyzed program is proposed as a simple and brief strategy to contribute to the control of the AIM.

Keywords: Music Education; Educational Psychology; Intervention; Anxiety; Singing, Professional Training.

Sumario. 1. Introducción. 2. Metodología. 3. Análisis de datos. 4. Resultados. 5. Discusión y conclusiones. 6. Referencias bibliográficas.

Cómo citar: Fernández-Granados, L.; Bonastre, C. (2021) La Ansiedad en la Interpretación Musical: programa de intervención en un coro, en Revista Electrónica Complutense de Investigación en Educación Musical 18, 49-60. http:// dx.doi.org/10.5209/reciem.68541

\section{Introducción}

La Ansiedad en la Interpretación Musical (en adelante, AIM), ha sido reconocida como un problema para los intérpretes pues se trata, en líneas generales, de una manifestación concreta de ansiedad que tiene que ver con el miedo a cometer un error o no ser capaz de controlar las acciones propias enfrente de otras personas (Iusca y Dafinoiub, 2012;

\footnotetext{
Consejería de Educación y Juventud de la Comunidad de Madrid

E-mail: lfernandezgranados@educa.madrid.org

https://orcid.org/0000-0002-2276-2511

2 Universidad Autónoma de Madrid (España)

E-mail: carolina.bonastre@uam.es

https://orcid.org/0000-0001-8632-5655
}

Rev. electrón. complut. inves. educ. music. 18, 2021: 49-60 
Papageorgi y Kopiez, 2012). El DSM-5 considera la ansiedad escénica como una especificación del trastorno de ansiedad social (Asociación Americana de Psiquiatría, 2014), no obstante, a pesar del incremento de investigación en las últimas décadas sobre la AIM, aún los límites del concepto y su diagnóstico permanecen un poco difusos (Kenny, 2011). En un estudio reciente sobre la relación entre la AIM y la ansiedad social no se aprecian evidencias de una conexión entre ambas, aunque sí parece que otros trastornos como el Trastorno de Ansiedad Generalizada pueden ejercer de mediadores entre la AIM y cualquier tipo de ansiedad (Wiedemann, Vogel, Voss, Nusseck y Hoyer, 2021), alineándose esta con la concepción de la AIM de Kenny (2016).

Según una reciente revisión sistemática de las investigaciones dirigidas a la intervención de la AIM en las últimas décadas, la tasa de prevalencia de vida en músicos varía entre $16.5 \%$ y $60 \%$, afectando aproximadamente a un tercio de los músicos (Fernholz et al., 2019) en algún momento. Advierten los investigadores de que es posible que la falta de consenso en cuanto a la conceptualización y la incertidumbre diagnóstica implique cierta ambigüedad que puede explicar el amplio rango de tasas de prevalencia.

Respecto a la terminología empleada, se aprecia un debate extendido en el tiempo donde unos autores usan indistintamente un término u otro: determinados investigadores justifican el uso de un concepto en concreto y otros manifiestan que esta discusión es poco relevante. Siguiendo a Steptoe (2001), consideramos más apropiado referirnos a este tipo de ansiedad concreta como AIM por las siguientes razones: (a) se refiere específicamente a las sensaciones experimentadas por músicos, a diferencia del miedo o pánico escénico que pueden sentir también actores, bailarines, conferenciantes o presentadores; (b) la AIM puede darse en diferentes entornos y no solo en el escenario (una clase o una audición, por ejemplo) porque depende más de la naturaleza evaluativa de la situación que de la presencia de público o no; (c) los términos "miedo" y "pánico" implican una alarma repentina, mientras que la AIM puede ser predecible y desarrollarse gradualmente antes de una ocasión importante; (d) el término no solo se limita al miedo que experimenta el músico, también influye en la interpretación, pues la calidad de la misma puede alterarse.

Kenny (2011) define el concepto apoyándose en la teoría de la triple vulnerabilidad de Barlow (2000, 2002), el marco teórico más influyente sobre la ansiedad en los últimos 20 años, que además se encuentra vinculado y está en el origen de las investigaciones actuales más importantes sobre los trastornos de ansiedad y los factores que le afectan, proporcionando de esta manera un marco teórico más sólido. De hecho, considera que muestra una combinación de síntomas afectivos, cognitivos, fisiológicos y conductuales y se puede producir una asociación de comorbilidad con "otros trastornos de ansiedad, en particular, la fobia social. Afecta a los músicos durante toda la vida y es al menos parcialmente independiente de los años de práctica y el nivel de logro musical” (p. 433). La amplitud de elementos que le influyen y las características específicas que le definen y diferencian de cualquier tipo de trastorno de ansiedad se abordan desde múltiples perspectivas, por tanto, existe cierta unanimidad al considerarlo un constructo multidimensional. El nivel de activación depende de la interacción de varios factores como la susceptibilidad del intérprete para experimentar ansiedad cuando se compromete a tocar o a cantar, la percepción de autoeficacia del músico (referido a la preparación, motivación, estrategias de afrontamiento, dificultad de la tarea, entre otras), las características específicas del entorno donde se produce la interpretación, con la influencia de parámetros como la presencia y el tipo de público, el grado de exposición percibido y las características del lugar (Papageorgi, Hallam y Welch, 2007; Papageorgi y Kopiez, 2012). Por tanto, la AIM es multifacética y conlleva elementos diversos del dominio fisiológico, cognitivo, motor y afectivo-emocional del funcionamiento humano (Clark y Beck, 2010; Emmons y Thomas, 2008; Yoshie, Kudo y Ohtsuki, 2008), y será a través de indicadores como los síntomas de cada componente por los que se podrá analizar sus niveles.

El componente fisiológico se caracteriza por la preparación y activación de los sistemas nerviosos autónomo y central y el sistema endocrino para responder frente a la situación y entre sus manifestaciones se encuentran las alteraciones del ritmo cardíaco y de la respiración, la transpiración, la tensión muscular, la falta de salivación, manos frías y/o sudorosas, necesidad urinaria frecuente, entre otras (Papageorgi y Kopiez, 2012; Yoshie et al., 2008). El componente cognitivo se identifica con el razonamiento, los métodos de afrontamiento de las situaciones o la forma de percibir la realidad. En los momentos de más ansiedad el razonamiento deductivo y las formas de pensamiento constructivas son menos accesibles y más lentas (Clark y Beck, 2010), generando consecuencias como la pérdida de concentración y atención, distracciones, fallos en la memoria o mala lectura de la partitura musical (Papageorgi y Kopiez, 2012). Se ha observado que la calidad de la interpretación se reduce si los músicos durante la actuación, además de experimentar síntomas de AIM, generan pensamientos y percepciones al respecto (Clark, Lisboa y Williamon, 2014).

Las conductas motoras se generan también como respuesta siendo las más básicas innatas y adaptativas para la supervivencia (ataque, huida o evitación). Destacan entre sus síntomas los temblores, los fallos en la ejecución, las modificaciones del tempo y la falta de coordinación motora (Papageorgi y Kopiez, 2012). El componente afectivo-emocional se identifica con los síntomas de tensión, aprensión subjetiva o pánico relacionado con la exposición en público, la preocupación, el miedo al fracaso, la inestabilidad emocional, la vulnerabilidad ante las críticas o los problemas en las relaciones sociales (Kobori, Yoshie, Kudo y Ohtsuki, 2011; Nagel, 2010; Ortiz, 2011a). El instrumento más habitualmente empleado para la evaluación de la AIM es el Kenny Music Performance Anxiety - Revised (K-MPAI R) (Kenny, 2009), que reúne sus elementos en tres grandes grupos: Early relationship context (contexto de las relaciones tempranas), Psychological vulnerability (vulnerabilidad psicológica) y Proximal performance concer$n s$ (preocupaciones asociadas a actuaciones cercanas) (Kenny, 2009). 
Con respecto al estado de la cuestión en España en la última década encontramos que la mayoría de estudios versan sobre cómo afecta la AIM en un grupo de edad o nivel de estudios concreto (Urruzola y Bernaras, 2020; Zarza-Alzugaray, Orejudo, Casanova y Aparicio-Moreno, 2017; Zarza-Alzugaray, Casanova y Orejudo, 2016a); sobre cómo afecta la AIM a determinados grupos, como por ejemplo, al alumnado de flauta travesera de varios conservatorios profesionales de Andalucía (Herrera, Manjón y Quiles, 2015); y sobre la relación de la AIM con otros constructos y otras cuestiones asociadas (González, Blanco-Piñeiro y Díaz-Pereira, 2018; Orejudo, Zarza-Alzugaray y Casanova, 2018; Orejudo, Zarza-Alzugaray, Casanova, Rodríguez-Ledo y Mazas, 2017; Zarza-Alzugaray, F. J., Casanova, O., y Orejudo, S. 2016b; Zarza-Alzugaray, Casanova-López y Robles-Rubio, 2016). También se registra una revisión sistemática acerca de la epidemiología y los tratamientos de la AIM (Ortiz 2011a, 2011b) y la adaptación española del instrumento más relevante para medir la AIM (Zarza-Alzugaray, Orejudo, Casanova, y Mazas, 2015). Solamente una investigación se dirige a comprobar la eficacia de diferentes técnicas y métodos de intervención psicopedagógicos en un estudio con cuatro estudiantes de Grado Superior (Viejo y Laucirica, 2016).

Por lo tanto, en la actualidad, aunque existen talleres o asignaturas destinadas a un área específica de la AIM, no se encuentran programas de intervención diseñados para tratarla globalmente. Las investigaciones sobre intervención incluidas en las revisiones (Fernholz et al., 2019; Kenny, 2005; Nagel, 2010; Ortiz, 2011b) indican la existencia de pocos tratamientos desde una perspectiva multidimensional y de la falta de efectividad adecuadamente probada. Entre las técnicas y métodos que mayor impacto han resultado sobre la AIM según las investigaciones realizadas están las relativas a la terapia cognitivo-conductual, pues en todas ha supuesto una reducción de AIM después de las intervenciones (Fernholz et al., 2019), como las visualizaciones, concentración en la respiración, la meditación, técnicas de relajación, así como la promoción de estilos de vida positivos como mayor actividad física, sueño adecuado, hábitos más saludables en general, la técnica Alexander, el yoga o una adecuada nutrición (Kenny, 2005; Nagel, 2010).

Desde un punto de vista preventivo, Ortiz (2011b) señala que es fundamental que desde el inicio de la formación musical exista una consonancia entre una pedagogía sólida (que considere el repertorio más adecuado a las habilidades de los estudiantes), un ambiente familiar favorable que permita el libre desarrollo de los niños y adolescentes y el aprendizaje de estrategias de autorregulación. A pesar de la creciente investigación en el campo de la interpretación, se evidencia una falta de estudios a nivel de aula donde los resultados beneficien a los estudiantes de música y que propicien cambios en la pedagogía de los docentes, un tipo de investigación construida desde y para la práctica.

El contexto de la intervención de este estudio surge de la colaboración de un coro universitario de Madrid con una compañía teatral en un espectáculo de la obra Carmina Burana de Carl Orff. Puesto que la mayoría de los componentes (profesorado, personal de la universidad, estudiantes universitarios, profesionales de otros campos vinculados a la universidad) no tenían formación musical previa, se diseñó de forma específica un programa de intervención breve sobre la AIM en función de las características del proyecto, la disponibilidad y el perfil de los participantes. Con sesiones de 15 minutos en las que se combinaban actividades de distinta naturaleza se facilitaría la adquisición de recursos y herramientas para la reducción de la ansiedad, la mejora de la presencia escénica y el disfrute de las 12 funciones musicales que componían el proyecto.

\subsection{Objetivos}

El objetivo principal consiste en diseñar, implementar y evaluar un programa de intervención que contemple los cuatro componentes: cognitivo, fisiológico, motor y afectivo-emocional. El segundo objetivo es aportar herramientas y recursos educativos para que los músicos puedan afrontar las interpretaciones musicales de la forma más adaptativa posible.

\section{Metodología}

Los métodos mixtos de investigación consisten en la complementariedad metodológica cuantitativa y cualitativa con la finalidad de favorecer una mayor comprensión acerca del objeto de estudio, aprovechando las fortalezas y minimizando las debilidades de los estudios de investigación (Johnson y Onwuegbuzie, 2004). Este estudio se adscribe al Diseño Explicativo Secuencial de métodos mixtos.

La primera fase presenta un diseño preexperimental con prueba pretest - postest en un solo grupo (intragrupo), donde el programa de intervención fue la variable independiente, y las variables dependientes fueron las dimensiones de vulnerabilidad psicológica, los pensamientos específicos, el factor contextual, el motor y fisiológico, y el afectivo-emocional. La fase cualitativa la componen el análisis de las preguntas abiertas y el grupo de discusión realizado tras la intervención.

\subsection{Muestra}

Los participantes fueron 46 cantantes de un coro de una universidad compuesto por un $45.7 \%$ de mujeres (media de edad $=42.25$, DT $=15.79$ ), y un $50 \%$ de hombres (media de edad $=48.00, \mathrm{DT}=12.72$ ), el $4.3 \%$ restante son datos perdidos. En su mayoría eran aficionados (65.2\%), aunque varios de sus integrantes combinaban sus profesiones o 
estudios universitarios con los estudios de grado elemental, profesional o superior (23.9\%), y profesionales de la música (10.9\%). El rango de edad oscilaba entre 18 y 71 años, siendo la media total de 45.33 años (DT=14.35).

\subsection{Instrumentos}

\section{1) Medidas cualitativas}

Se incluyeron dos preguntas abiertas en el cuestionario vinculadas a los objetivos de la investigación: ¿cuántas veces al año cantas/tocas ante público como solista o en un grupo reducido (cuarteto, quinteto)? y explica brevemente cómo controlas o afrontas la ansiedad antes y durante una interpretación musical. Se codificaron las respuestas reduciéndolas y categorizándolas mediante códigos. Además, se empleó el grupo de discusión posteriormente con varios de los participantes para una mejor comprensión de la realidad estudiada. Dado que las opiniones y percepciones de los sujetos se retroalimentan, aumenta en consecuencia la calidad y cantidad de los datos pese a la carga de subjetividad (McMillan y Schumacher, 2011).

\section{2) Medidas cuantitativas}

Las intervenciones psicoterapéuticas para la AIM precisan abordar de forma global los componentes específicos la ansiedad. El inventario K-MPAI-R (Kenny, 2009) aborda la evaluación de los componentes de la ansiedad postulados por Barlow (2000). No obstante, se hace necesario incluir un componente más asociado a la AIM sin el cual no podría entenderse toda su complejidad, el componente afectivo-emocional (Abril, 2007; Emmons y Thomas, 2008; Osborne y Kenny, 2008). En este estudio se proponen cinco dimensiones con la intención de evaluar y conocer más en profundidad el funcionamiento de los componentes de la AIM. Relacionado con la vulnerabilidad psicológica específica de Barlow $(2000,2002)$ se encuentran la Vulnerabilidad Psicológica (VP), categoría también propuesta por Kenny (2009); los Pensamientos Específicos (PE), también dentro del grupo de Proximal performance concerns (Kenny, 2009, al igual que la dimensión Afectivo- Emocional (AE), y Motor y Fisiológico (MF). La dimensión Contextual (C), se asocia a las experiencias tempranas de la vulnerabilidad psicológica generalizada de Barlow (2000, 2002), y a Early relationship context (Kenny, 2009). El cumplimiento de los objetivos de esta investigación precisa una serie de ajustes y de adecuación del instrumento, así pues, se parte de la validación para la población española del K-MPAI realizada por Zarza-Alzugaray et al. (2015), y se aprueba la reestructuración mediante un comité multidisciplinar de expertos.

El cuestionario final cuenta con una buena consistencia interna $(\alpha=.92)$, así como las escalas que, formadas por 50 ítems, se relacionan y evalúan las dimensiones de la AIM: Vulnerabilidad Psicológica $(\alpha=.76)$, Pensamientos Específicos $(\alpha=.87)$, Dimensión Contextual $(\alpha=.63)$, Motor y Fisiológico $(\alpha=.79)$ y Afectivo-Emocional $(\alpha=.83)$. Se responden en función del grado de acuerdo en una escala tipo Likert de siete puntos, siendo el 1 (total desacuerdo o nada) y el 7 (totalmente de acuerdo o mucho).

\subsection{Procedimiento}

Se establecieron las siguientes etapas procedimentales: (1) se diseñó un programa de intervención ajustado a las necesidades del grupo mediante la colaboración de varios expertos y se consensuó con el director del coro, estableciendo conjuntamente el cronograma de las sesiones; (2) en la sesión inicial se presentó el proyecto, a la persona que impartiría las sesiones, y firmaron un consentimiento informado; (3) se explicó cómo responder al cuestionario y a las preguntas en esa sesión inicial y se resolvieron las dudas; (4) se aplicó el programa en 8 sesiones, además de la inicial y la final; (5) después de la intervención se administraron los mismos instrumentos que en la prueba previa; y (6) días después de la intervención se reunieron diez participantes conformando un grupo de discusión para debatir sobre cuestiones relativas a la experiencia vivida (interpretación, exposición en público, síntomas de la AIM o ambiente profesional, entre otras).

El programa se implementó durante las dos semanas de ensayos generales y funciones en el teatro, en un espacio que permitía la movilidad del grupo. Las sesiones se distribuyeron en dos bloques de cuatro sesiones de aproximadamente 15 minutos cada una, aunque hubo reajustes de actividades o de duración dependiendo de las necesidades técnicas de cada día. Se contó con dos días a la mitad del programa para que los participantes utilizaran autónomamente los recursos y herramientas. La sesión inicial coincidió con el ensayo pre-general y la final, con el día de la última función. Estas sesiones evaluativas tuvieron una duración de 30 minutos para introducir adecuadamente el programa y sus objetivos, y para completar el cuestionario y las preguntas. Incluimos a continuación una explicación detallada de las sesiones que componen el programa de intervención, así como la referencia a los métodos, técnicas y recursos empleados (Tabla 1). 
Tabla 1. Aspectos trabajados durante las sesiones.

\begin{tabular}{|c|c|}
\hline Función/Sesión del programa & Aspecto trabajado \\
\hline Ensayo pre-general / Sesión 0 & $\begin{array}{l}\text { Introducción al programa, firma del consentimiento in- } \\
\text { formado, realización de la evaluación inicial. }\end{array}$ \\
\hline Ensayo general / Sesión 1 & Psicoterapia grupal, ejercicio de visualización. \\
\hline Función 1 / Sesión 2 & Conciencia corporal, dinámica de grupo: empaste vocal. \\
\hline Función 2 / Sesión 3 & $\begin{array}{l}\text { Ejercicios de calentamiento vocal con expresión corpo- } \\
\text { ral, estiramientos y visualización. }\end{array}$ \\
\hline Función 3-4 / Sesión 4 & Control y conciencia corporal, respiración y relajación. \\
\hline Función 5 & - \\
\hline Función 6 & - \\
\hline Función 7 / Sesión 5 & TRP. \\
\hline Función 8 / Sesión 6 & Concentración y desinhibición, TRP. \\
\hline Función 9 / Sesión 7 & Exposición y psicoterapia, TRP. \\
\hline Función 10-11 / Sesión 8 & $\begin{array}{l}\text { Actividades de refuerzo de la autoestima, confianza gru- } \\
\text { pal y TRP. }\end{array}$ \\
\hline Función 12/Sesión final & Realización de la evaluación final. \\
\hline
\end{tabular}

Nota. TRP: Técnica de Relajación Progresiva.

En la sesión 0 se explicó a los participantes en qué consistía el programa, firmaron el consentimiento informado (5 minutos), y realizaron la evaluación inicial (25 minutos). La sesión 1 estaba compuesta por una actividad de psicoterapia grupal (10 minutos) basada en las terapias cognitivas para los trastornos de ansiedad (Clark y Beck, 2010) que consistía en una puesta en común acerca de cómo está viviendo la experiencia cada uno. También se explicó y se realizó una visualización guiada (10 minutos); en este caso se relacionó con la experiencia del estreno del espectáculo, empleándose para ello una adaptación de las pautas de Bados y García (2011). En la sesión 2 se trabajó la relajación (10 minutos) para favorecer la conciencia de la respiración y de las sensaciones corporales a través de movimientos lentos, inspirada en el método Feldenkrais (Feldenkrais, 1992). Los participantes por parejas fueron indicando a su compañero diversas partes del cuerpo para que se concentraran en la relajación de las mismas, y comprobándolo posteriormente, moviendo de forma delicada esa parte. Se finalizaba con un ejercicio en corro para trabajar el empaste vocal (5 minutos), los participantes colocaban una mano sobre el cuello de los compañeros situados a los lados y con los ojos cerrados. El guía de la actividad pasaba por detrás del corro e indicaba a un participante con un contacto en el hombro que debía emitir un sonido muy suave, transmitiendo tanto el sonido como la vibración a sus compañeros, que a su vez se lo transmitirían a los que tenían al lado (aportación del Experto 1).

La sesión 3 incluía dos actividades de calentamiento vocal con expresión vocal. La primera (4 minutos) consistía en pedir a los participantes que se movieran por el espacio y cuando quisieran, que comenzaran a emitir sonidos vocalmente, fijándose en las señas que iba realizando el guía para seguir moviéndose igual pero sin emitir sonido alguno (aportación del Experto 1). Para la segunda actividad (6 minutos) se indicaba un fragmento concreto de la obra que estaban trabajando, puesto que sobre ese fragmento se irían introduciendo cambios en la vocalización con las indicaciones de quien guiaba el ejercicio. Con los gestos de su mano derecha indicaría los matices y con los de la izquierda, el nivel de nasalidad - twang o voz impostada y redonda -. La última actividad de la sesión (5 minutos) se inspiraba en el trabajo de Koeppen (1974) y consistía en realizar una serie de estiramientos lentos a través de la representación de la vida de un árbol mediante la expresión corporal. En la sesión 4 se trabajó, en primer lugar, la conciencia corporal y la concentración relajada (7 minutos) siguiendo las pautas de García (2013) en su obra para músicos sobre la Técnica Alexander. Se trataba de prestar atención a las diferentes zonas corporales aumentando la conciencia que se tiene sobre ellas y llevándolas a la relajación. Por otro lado, y siguiendo al mismo autor, se introdujo un ejercicio para aumentar la conciencia de la respiración manteniendo el estado de relajación alcanzado con el ejercicio anterior (8 minutos). En la sesión 5 se presentó la Técnica de Relajación Progresiva (en adelante, TRP) (15 minutos), explicando en qué consiste la técnica original de Jacobson, sus objetivos y pautas previas, aunque para el ejercicio se empleó la adaptación de Payne (1996). El objetivo final consiste en relajarse en 20-30 segundos y trasferir esta técnica a las situaciones más estresantes o de mayor presión, mediante ciclos de tensión-distensión por grupos musculares.

En la sesión 6 se realizó un ejercicio de concentración y de desinhibición ( 8 minutos) que consistía en imitar por parejas los movimientos en espejo del otro, aumentando progresivamente las series hasta equivocarse (aportación del Experto 2). Después se recordó en qué consistía la TRP, los pasos que se deben seguir y se realizó una práctica (7 minutos). La sesión 7 comenzó con una actividad de exposición pasiva ante los demás (10 minutos) que consistía en colocar una silla frente al grupo, que se encontraba sentado en el suelo. Por turnos y sin decir nada, uno a uno se iban sentando para observar a los compañeros que estaban sentados en el suelo y a su vez, ser observado. Después del ejercicio, los participantes intercambiaban impresiones sobre sus sensaciones al ser observados 
(aportación del Experto 2). La sesión concluía con la práctica de la TRP (5 minutos) para reducir progresivamente los tiempos necesarios para relajar los grupos musculares e interiorizar la técnica. La sesión 8 estaba compuesta por una actividad de autoestima y confianza grupal (10 minutos) que implicaba elogiarse a uno mismo ante otros respondiendo a una serie de consignas (Navarro, 2018). Las cuestiones consistían en decir: a) dos atributos físicos que me agradan de mí mismo (cambiaban de pareja); b) dos cualidades de personalidad que me agradan de mí mismo (cambiaban de pareja); c) una capacidad o habilidad que me agrada de mí mismo (cambiaban de pareja); y d) destaca una cualidad de tu compañero o compañera. Después, se procedía a la práctica de la segunda fase de la TRP (5 minutos) donde se eliminaba el tiempo de tensión de los músculos y queda solo la relajación. Se les recordaba que deben practicar la relajación progresiva por fases para reducir los tiempos y aplicarlo en situaciones de estrés con rapidez y discreción.

En la sesión final se realizaba la evaluación final (30 minutos), recordando previamente las instrucciones para completar correctamente el cuestionario. Una semana después de la última representación de la obra en el teatro, se realizó en la universidad la sesión del grupo de discusión (40 minutos aproximadamente). Participaron diez miembros del coro, quienes comentaron sus impresiones sobre la experiencia en una charla distendida.

\section{Análisis de datos}

Los cambios producidos en las variables dependientes se observaron mediante los análisis descriptivos (medias y desviaciones típicas) antes y después de la intervención, además de analizar los descriptivos de los ítems que componen las escalas. Se realizaron análisis de varianza (ANOVA) y pruebas $t$ de Student en la diferencia pretest-postest en cuanto al perfil del participante, respectivamente. Se calcula el tamaño del efecto (estimando los valores de d de Cohen como pequeño <.30; alrededor de .50, efecto moderado; grande $\geq .80$; Cohen, 1988) con un $95 \%$ de intervalo de confianza en las diferencias pretest-postest de cada variable.

Los análisis cualitativos de las preguntas abiertas se realizaron categorizando las respuestas según el perfil de cada grupo con respecto a la música (estudiantes, profesionales y aficionados) para la pregunta relacionada con exposición en público anualmente; y para la relativa a los métodos de afrontamiento de la ansiedad en las actuaciones, las respuestas se clasifican según los cuatro componentes de la AIM (cognitivo, fisiológico, motor y afectivo-emocional), además de tres categorías correspondientes a la ausencia de ansiedad, al desconocimiento, y a la no gestión de la situación. El grupo de discusión se analiza mediante la coocurrencia de códigos y el establecimiento de redes.

Para los análisis se emplearon el paquete estadístico SPSS versión 20.0, y el ATLAS.ti versión 8.3.1.

\section{Resultados}

Para evaluar los efectos del programa en las dimensiones de la AIM (Vulnerabilidad Psicológica, Pensamientos Específicos, Dimensión Contextual, Motor y Fisiológico y Afectivo-Emocional), se realizó en primer lugar el análisis descriptivo (medias y desviaciones típicas) de las escalas en la prueba previa y en la posterior, así como la diferencia de las puntuaciones en ambas condiciones (Tabla 1). Las diferencias entre las dos condiciones mostraron que las puntuaciones promedio de los participantes, aunque no fueron estadísticamente significativas, disminuyeron, siendo las dimensiones de Motor y Fisiológico $(\mathrm{M}=-0.34)$ y Afectivo-Emocional $(\mathrm{M}=-0.32)$ donde se aprecia más esa mejora. Para un análisis más exhaustivo y propiciar la comprensión de las causas con la metodología cualitativa, se observaron los cambios producidos entre ambas condiciones en cada elemento de las escalas, encontrando diferencias estadísticamente significativas en elementos de Vulnerabilidad Psicológica, Pensamientos Específicos y Motor y Fisiológico (Tabla 2).

Tabla 2. Resultados descriptivos (medias y desviación típica) pretest y postest de las dimensiones e ítems estadísticamente significativos.

\begin{tabular}{lcccc}
\hline & \multicolumn{2}{c}{ Postest } & \multicolumn{2}{c}{ Pretest } \\
\hline Ítem & M & DT & M & DT \\
\hline Dimensión Vulnerabilidad Psicológica (VP) & 2.74 & 0.94 & 2.48 & 0.82 \\
Dimensión Pensamientos Específicos (PE) & 3.31 & 0.94 & 3.09 & 0.76 \\
Dimensión Contextual & 3.84 & 1.09 & 3.69 & 1.03 \\
Dimensión Motor y Fisiológico (MF) & 3.08 & 0.97 & 2.74 & 0.91 \\
Dimensión Afectivo-Emocional (AE) & 3.44 & 1.31 & 3.12 & 1.20 \\
VP2 - Raramente siento que controlo mi vida. & 2.93 & 1.84 & $1.95^{* *}$ & 1.15 \\
PE3 - No tengo ansiedad cuando me preparo mucho una & 3.80 & 1.75 & $3.09^{*}$ & 1.75 \\
prueba o un concierto. & & & &
\end{tabular}


PE6 - Antes de los conciertos dudo de si haré una buena interpretación.

PE10 - Pensar en que estoy siendo evaluado interfiere en mi interpretación.

MF2 - Durante un concierto o prueba, experimento temblores en alguna parte del cuerpo, como en las extremidades, en el labio o en la cara.

MF9 - Durante un concierto o prueba, me aumenta considerablemente el ritmo cardíaco.

$\begin{array}{llll}4.41 & 1.92 & 3.77 * & 1.67 \\ 5.00 & 1.45 & 4.23 * & 1.79 \\ 3.24 & 2.12 & 2.45 * & 1.58 \\ 4.51 & 1.62 & 3.70 * & 1.81\end{array}$

Nota. $N$ : total de la muestra. M: media. DT: desviación típica. ${ }^{*} p<.05,{ }^{*} p<.01, * * * p<.001$.

En segundo lugar, dado que las diferencias entre la condición previa y la posterior resultaron estadísticamente significativas en determinados ítems pero fueron leves y no significativas en cuanto a las dimensiones de forma global, consideramos oportuno estimar el tamaño del efecto de Cohen (1988). A pesar de las indicaciones del autor de evitar la interpretación por niveles del tamaño del efecto, parece existir cierto consenso con respecto a unos puntos de corte que permiten su interpretación (Rivera, 2017). Podríamos estimar como pequeño el tamaño del efecto en todas las dimensiones de la AIM, exceptuando la dimensión Motor y Fisiológico, cuyo tamaño del efecto resultó moderado (Tabla 3).

En tercer lugar, empleamos los análisis de ANOVAs univariados para estimar la asociación entre el perfil de los componentes del coro (estudiantes, aficionados y profesionales) y las variables dependientes, además de comparar las puntuaciones de la diferencia entre las pruebas preliminares y las posteriores siguiendo el mismo criterio (Tabla 3 ), encontrando que los resultados de Vulnerabilidad Psicológica $(\mathrm{F}(2,33)=.12 ; p=.89)$, Pensamientos Específicos $(\mathrm{F}(2,33)=2.18 ; p=.13)$, Dimensión Contextual $(\mathrm{F}(2,39)=.13 ; p=.88)$, Motor y Fisiológico $(\mathrm{F}(2,36)=.40 ; p=.96), \mathrm{y}$ Afectivo-Emocional $(\mathrm{F}(2,40)=.70 ; p=.50)$, no son estadísticamente significativos y por tanto, no se encuentran asociados el perfil y las puntuaciones de las dimensiones.

Tabla 3. Resultados del análisis de varianza (ANOVA) respecto al perfil y tamaño del efecto de las variables dependientes.

\begin{tabular}{llll}
\hline & \multicolumn{3}{c}{ Diferencias Pretest-Postest } \\
\hline Vulnerabilidad Psicológica & F & $\boldsymbol{p}$ & $\boldsymbol{d}$ \\
Pensamientos Específicos & 0.12 & .89 & 0.30 \\
Dimensión Contextual & 2.18 & .13 & 0.26 \\
Motor y Fisiológico & 0.13 & .88 & 0.13 \\
Afectivo-Emocional & 0.04 & .96 & 0.35 \\
\hline
\end{tabular}

Nota. $d=$ Tamaño del Efecto de Cohen. $n=46$.

Respecto a los análisis cualitativos, se analizaron por un lado las preguntas abiertas, y por otro, el grupo de discusión. En cuanto a las preguntas abiertas, ante la cuestión aproximadamente, ¿cuántas veces al año cantas/tocas ante público como solista o en un grupo reducido (cuarteto, quinteto,...)? La media de veces anuales de los estudiantes fue de 4.6 (DT=2.76); los profesionales, una media de 6.3 veces $(\mathrm{DT}=3.30)$; y la media de los aficionados, desciende a 3.3 veces por año $(\mathrm{DT}=1.20)$. La cantidad de veces que actúan ante un público no parece que difiera notablemente a pesar de la naturaleza de cada perfil, por lo que es probable que se deba a una percepción diferente de la pregunta. De este modo, probablemente los estudiantes contabilizan sus audiciones en su centro de estudios por las que son habitualmente evaluados, y en casos puntuales, añaden audiciones o conciertos externos a la cuenta de conciertos anuales; los aficionados es posible que contasen los conciertos de grupos numerosos; y finalmente, los profesionales incluirían solo el número de conciertos remunerados, idea que se confirma con la afirmación de un cantante, que decía que el número de conciertos varía en función del mercado de cada año. En cuanto a los estudiantes, resultan muy poco numerosas las oportunidades de tocar o cantar en público cuando su enseñanza está dirigida a alcanzar una cualificación profesional. Por tanto, la incoherencia encontrada en los resultados puede deberse a que las respuestas han atendido a criterios diferentes según la experiencia, visión y contexto del corista.

Para la segunda cuestión se categorizaron las respuestas atendiendo a los cuatro componentes de la AIM (afectivo-emocional, cognitivo, fisiológico y motor). Se trata de la pregunta concerniente a los métodos de afrontamiento: Explica brevemente cómo controlas o afrontas la ansiedad antes y durante una interpretación musical.

Con respecto al componente afectivo-emocional se encuentra en la evaluación previa que varias citas aluden a la necesidad de un tiempo en soledad y retraimiento para concienciarse o relajarse. Es una necesidad comprensible porque compartían un espacio pequeño unos cincuenta cantantes donde inconscientemente se retroalimentaba el estado emocional, normalmente el nerviosismo. Con respecto a las cuestiones relativas a los pensamientos, también identi- 
ficadas con el componente cognitivo, poseen a su vez un matiz afectivo-emocional porque implican una mejora del estado emocional de la persona que utiliza el recurso. Por ejemplo, "pensar positivamente" (Participante 7) ${ }^{3}$ supone un refuerzo anímico, al igual que cuando expresaban que su recurso es el disfrute o "...un sentimiento de ilusión y alegría ante el concierto" (P16), pues se intentaba provocar con ello un estado de bienestar. Desde las afirmaciones como "no preocuparme por lo que piensen los demás" (P29) y "nada es demasiado importante y que no me va a juzgar nadie" (P20), se intenta alejar la presión de la evaluación externa para evitar desestabilizarse. Ciertos hábitos les proporcionaban tranquilidad y concentración, como las conductas motoras de aislamiento, la evitación de pensamientos o del repaso de la partitura, además de la práctica de ejercicios de visualización o controlando y dirigiendo los pensamientos hacia un punto que les aportaba seguridad, tal y como reflejan los comentarios "pienso en mí misma como persona solista" (P24) o "pienso que va a salir bien" (P13).

En los métodos relacionados con el componente fisiológico incluyeron acciones positivas que generan bienestar físico como la hidratación o el deporte, pero también algunas perjudiciales como la ingesta de ansiolíticos o el tabaco, sustancias que disminuyen de forma aparente y rápida los síntomas físicos de la ansiedad, aunque de forma pasiva y con consecuencias sobre la salud. Se aprecia también en los comentarios el desconocimiento o la falta de los recursos y técnicas antes de realizarse el programa de intervención, puesto que se registran expresiones tales como "no tengo un método para controlar la ansiedad" (P18), o "al principio sí me estresaba" (P10), donde se evidenciaba la incapacidad de una correcta gestión debido a la ausencia de recursos para afrontarla. Este tipo de pensamientos de incapacidad o de ineficacia en el manejo de la AIM se relaciona con los resultados de un estudio sobre estados emocionales de un grupo de adolescentes músicos antes de tocar en público, que a su vez correlacionaban con niveles altos y moderados de AIM (Kaleńska-Rodzaj, 2019). La cantidad de respuestas relativas a recursos o herramientas disponibles es similar antes y después de la intervención, sin embargo, la expresión de las ideas resulta más elaborada, deduciéndose de este hecho que se produjo un mejor entendimiento del funcionamiento de las mismas y que se han entrenado en su uso.

En la evaluación posterior a la intervención aludieron menos a cuestiones afectivas y mencionaron más cuestiones de espacio y tiempo en soledad, como con la expresión "distanciamiento del momento" (P17), que implica tanto un estado físico como una reducción de la intensidad emocional. También incrementaron las referencias a la seguridad en uno mismo como consecuencia de la práctica continuada, como se aprecia en verbalizaciones como "prestándome a cantar todo lo que pueda para ver si se vuelve una cosa [el hecho de actuar en público] normal" (P29), y el incremento del trabajo: “estudiando lo suficiente y llevándolo bien preparado" (P36). Los cantantes consideraron importante una buena preparación, puesto que les aporta seguridad un adecuado estudio y prestar "atención al trabajo" (P5), habituándose a la situación y normalizándola para convertirla en una ventaja psicológica sobre el momento. En cuanto al grupo de discusión, el análisis de la cantidad de citas en función de los códigos establecidos indica que la frecuencia de las menciones relativas a las manifestaciones de AIM, de forma explícita o implícita, son elevadas.

Se establecieron distintas redes de códigos tras el análisis, siendo la más relevante la que contempla los síntomas de la AIM. Estos interactúan y en ocasiones se superponen, por tanto, es complicado delimitar los componentes con los que se identifican. Los coristas vivieron subidas y bajadas emocionales constantes e intensas durante las semanas de ensayos y de los pases del espectáculo, por este motivo se registran más citas en el componente afectivo-emocional que del resto. Las sensaciones más significativas, dadas las numerosas repeticiones en las respuestas, son la ilusión, la energía y la motivación que tenían antes, durante y después del proyecto, pues contaban las experiencias entre sonrisas. Se sintieron satisfechos con el trabajo hecho, con haber cumplido el compromiso y muy contentos con la experiencia a la que consideraban un reto.

También se generaron emociones de tensión y estrés dada la intensidad del proyecto. Los síntomas cognitivos afectan en gran medida a los músicos puesto que el poder de los pensamientos condiciona los movimientos, la forma de comportarse y de enfrentarse a las situaciones. La preocupación que sentían los cantantes por hacer el trabajo correctamente les llevaba a estados de gran concentración mantenida durante todos esos días, como afirmaba una de las participantes "esta sensación de cada día necesitar mejorar y tomármelo muy en serio y no sé, eso tiene una responsabilidad también muy..." (P7). Compartieron comentarios donde evidenciaban la presencia de pensamientos negativos, catastróficos y dudas, sin embargo, después de tantos ensayos y funciones comprobaron que es factible tener un buen desempeño a pesar de la falta de formación específica, solo que implicaba un esfuerzo mayor. Este hecho generaba una gran satisfacción entre los coristas y se visibilizaba con expresiones del tipo: "mira, lo he hecho, lo he hecho dentro de mis posibilidades que creía, que creo que son limitadas, y el trabajo está bien" (P8).

El componente fisiológico es más sencillo de reconocer porque muchos de sus síntomas se aprecian visualmente, aunque a la vez son difíciles de gestionar porque están más interiorizados y arraigados. Los más habituales fueron el cansancio, los dolores de espalda, el sueño, las taquicardias y las tensiones. Con respecto a las conductas motoras, la ansiedad provocó un aumento de críticas tanto hacia uno mismo como a los demás o a lo que les rodea, la evitación de las situaciones estresantes o manifestaciones externas como temblores, o internas como inquietud. Las que más se identificaron en el grupo fueron el cansancio, los fallos que tuvieron, y el nerviosismo que se manifestaba en la alteración del sueño y en la sensación de aceleración.

En adelante se señalarán las intervenciones de los participantes con su número asignado. 


\section{Discusión y conclusiones}

Diversos investigadores han mostrado que los factores afectivo-emocionales y cognitivos son los predominantes en la AIM y que tienen mayor impacto sobre la interpretación musical y la autoconfianza (Osborne y Kenny, 2008; Yoshie, Kudo, Murakoshi y Ohtsuki, 2009), resultados que se sitúan en la línea de la gran preocupación percibida en los coristas por hacer bien su trabajo y estar a la altura de un espectáculo profesional. Entre las respuestas de una evaluación y otra observamos que los procesos afectivo-emocionales se tratan de forma más operativa en esta última, probablemente debido a que después de semanas de funciones consiguieran interiorizar esas ideas vinculadas a las emociones y se centraran en la autoconfianza. Se encuentra además que el trabajo de la reestructuración cognitiva (la estrategia cognitiva de relativizar) que a pesar de no trabajarse explícitamente, sí se practicó a través de la visualización, quedó incorporada a los recursos de los participantes.

El componente cognitivo fue el segundo componente con el que los coristas se implicaron más, dato que cobra sentido al ser la dimensión de Pensamientos Específicos la que más diferencias estadísticamente significativas presentó en sus ítems tras la intervención. Se evidenció un perfeccionamiento en el nivel de control sobre los pensamientos, pues practicaron la técnica de la detención de los pensamientos al aislarse y frenar cada pensamiento negativo o destructivo. Ese aislamiento tuvo beneficios también a nivel afectivo-emocional al no contagiarse del nerviosismo ajeno y centrarse en la relajación y conexión consigo mismo, logrando un estado de bienestar y calma necesarios para afrontar adecuadamente la función.

La mayoría de técnicas empleadas durante esas semanas se relacionaban con el componente fisiológico, destacando la relajación, los ejercicios de respiración y conciencia corporal o meditación. A nivel motor los recursos empleados fueron la habituación a situaciones estresantes debido a los múltiples ensayos y conciertos, la rigurosa preparación previa y también conductas relativas a la puntualidad. Estos resultados se relacionaron directamente con la mejora de puntuaciones de la escala Motor y Fisiológico, la que mejores resultados obtuvo de forma general. Resulta beneficioso y positivo el cambio de hábitos al respecto, pues en la evaluación posterior al menos ya no se menciona el consumo de tabaco o ansiolíticos para ayudar a afrontar la situación. El uso (y probablemente, abuso) de sustancias y medicación es un problema presente en la realidad de los músicos y cantantes a todos los niveles, incluidos los músicos de orquestas profesionales (Cohen y Bodner, 2019; Kenny, Driscoll y Ackermann, 2014).

Como si de una desensibilización sistemática se tratara, las semanas de ensayos previos del coro solo, los ensayos intensivos con la compañía y finalmente, las representaciones, supusieron una exposición progresiva al "estímulo fóbico". Al habituarse a la situación esta pierde su efecto sobre los implicados, además de posiblemente encontrar elementos satisfactorios que pueden actuar como refuerzo positivo, como la mejora de las relaciones sociales, la buena acogida del público, el reconocimiento de los profesionales y de los familiares, reemplazando la respuesta condicionada de miedo y ansiedad por otra más adaptativa.

En relación a la influencia del perfil o del grado de estudio de los participantes, no se apreciaron diferencias significativas en función de si se trataba de estudiantes, profesionales o aficionados. Esta circunstancia puede indicar, acorde a Kenny (2011), que la presencia de AIM no es inherente a la experiencia del músico dado que también se manifiesta entre músicos de orquestas profesionales como muestran investigaciones realizadas al respecto (véase James, 1998).

Nagel (2010) acertadamente transmite la idea de que la interpretación o la carrera musical sin miedos o ansiedad en ninguna circunstancia no es una realidad, aunque se debe luchar por el desarrollo de una capacidad de apreciar y aceptar las habilidades y las limitaciones propias dentro y fuera del escenario para favorecer el disfrute de las actuaciones musicales. Encontramos que el programa de intervención tuvo efectos positivos en la reducción de los niveles de AIM, así como mejoras en cuestiones de presencia escénica y del aprendizaje de métodos, técnicas y recursos que facilitaron la adaptación del grupo a los niveles de rendimiento propios del trabajo profesional propuesto en la colaboración, resultando una experiencia gratificante. No obstante, debido a las limitaciones que se exponen a continuación, es necesario interpretar con prudencia los resultados obtenidos.

Como limitaciones principales del estudio observamos que la elección del diseño preexperimental de un solo grupo con pretest y postest supone una dificultad para realizar deducciones causales (McMillan y Schumacher, 2011), por lo que para futuras investigaciones sería idóneo la inclusión de un grupo de control con el que comparar los resultados y establecer relaciones causales más consolidadas, del mismo modo que considerar el uso de otras técnicas de investigación que aporten nuevos resultados para poder atribuir mejor las diferencias significativas a la implementación del programa de intervención. Además, aunque las intervenciones breves tienen una fiabilidad demostrada (OMS, 2011; Yeager et al., 2016) sería recomendable para futuros trabajos tanto realizar una intervención de mayor duración para estudiar los resultados, como incluir un seguimiento para verificar el mantenimiento o la estabilidad del cambio producido por la intervención. Asimismo, se considera fundamental el desarrollo y la validación de un instrumento para la población española de músicos que contemple la evaluación de los cuatro componentes de la AIM.

De forma más dirigida al ámbito pedagógico, en líneas de investigación y de intervención futuras es necesario apostar por la prevención de la AIM de la forma más natural posible, y sobre todo en principiantes puesto que la AIM suele comenzar a gestarse en las primeras etapas del aprendizaje de la música (véase Fehm y Schmidt, 2006; Kenny y Osborne, 2006; Ryan, 2004, 2005). Promover que los docentes utilicen los recursos empleados en este programa en el aula puede favorecer que los alumnos mejoren aspectos no sólo relacionados con el propio proceso de enseñan- 
za-aprendizaje y de afrontamiento a ciertas situaciones, y también puede aportar mejoras en el propio desarrollo y crecimiento personal del alumnado.

Por tanto, las propuestas que consideramos más adecuadas para abordar las cuestiones planteadas desde contextos educativos musicales serían: la sistematización de estrategias preventivas, la consolidación de las intervenciones tanto de forma explícita (a través de programas, talleres u optativas) como de forma implícita (desde la pedagogía de los docentes), y mediante la implicación en el proceso de familiares, profesores, especialistas en orientación educativa y demás miembros involucrados, todo ello para minimizar los niveles de la AIM de los músicos.

\section{Referencias bibliográficas}

Abril, C. R. (2007). I have a voice but I just can't sing: A narrative investigation of singing and social anxiety. Music Education Research, 9(1), 1-15. https://doi.org/10.1080/14613800601127494

Asociación Americana de Psiquiatría-APA (2014). Manual Diagnóstico y Estadístico de los Trastornos Mentales, (5a Ed.) Madrid, España: Editorial Médica Panamericana.

Bados, A. y García, G. E. (2011). Técnicas de exposición. Departamento de personalidad, evaluación y tratamientos psicológicos. Barcelona, España: Universidad de Barcelona. Recuperado de: http://diposit.ub.edu/dspace/bitstream/2445/18403/1/T\%C3\%A9cnicas\%20de\%20Exposici\%C3\%B3n\%202011.pdf

Barlow, D. H. (2000). Unraveling the mysteries of anxiety and its disorders from the perspective of emotion theory. American psychologist, 55(11), 1247. https://doi.org/10.1037/0003-066X.55.11.1247

Barlow, D. H. (2002). Anxiety and its disorders: the nature and treatment of anxiety and panic (2a ed.). Nueva York, EEUU: Guilford Press. https://doi.org/10.1002/smi.941

Clark, D. A. y Beck, A. T. (2010). Terapia cognitiva para trastornos de ansiedad. Bilbao, España: Descleé de Brouwer.

Clark, T., Lisboa, T., y Williamon, A. (2014). An investigation into musicians' thoughts and perceptions during performance. Research Studies in Music Education, 36(1), 19-37. https://doi.org/10.1177/1321103X14523531

Cohen, J. (1988). Statistical Power Analysis for the Behavioral Sciences (2d ed.). Hillsdale, EEUU: Lawrence Erlbaum. https:// doi.org/10.4324/9780203771587

Cohen, S., y Bodner, E. (2019). The relationship between flow and music performance anxiety amongst professional classical orchestral musicians. Psychology of Music, 47(3), 420-435. https://doi.org/10.1177/0305735618754689

Emmons, S. y Thomas, A. (2008). Voice pedagogy: Understanding performance anxiety. Journal of Singing-The Official Journal of the National Association of Teachers of Singing, 64(4), 461-465.

Fehm, L., y Schmidt, K. (2006). Performance anxiety in gifted adolescent musicians. Journal of anxiety disorders, 20(1), 98-109. https://doi.org/10.1016/j.janxdis.2004.11.011

Feldenkrais, M. (1992). Autoconciencia por el movimiento: ejercicios para el desarrollo personal. Barcelona: Paidós.

Fernholz, I., Mumm, J. L. M., Plag, J., Noeres, K., Rotter, G., Willich, S. N., ... y Schmidt, A. (2019). Performance anxiety in professional musicians: a systematic review on prevalence, risk factors and clinical treatment effects. Psychological medicine, 49(14), 2287-2306. https://doi.org/10.1017/S0033291719001910

García, R. (2013). Técnica Alexander para músicos: La "zona de confort": salud y equilibrio en la música. Barcelona, España: Robin Book.

González,A.,Blanco-Piñeiro,P.,yDíaz-Pereira,M.P.(2018).Musicperformanceanxiety:Exploring structural relations withself-efficacy, boost, and self-rated performance. Psychology of Music, 46(6), 831-847. https://doi.org/10.1177/0305735617727822

Herrera T. L., Manjón G. J., y Quiles, O.L. (2015). Ansiedad escénica musical en alumnos de flauta travesera de conservatorio. Revista Mexicana de Psicología, 32(2), 169-181. https://doi.org/10.23923/rpye2020.01.184

Iusca, D., y Dafinoiu, I. (2012). Performance anxiety and musical level of undergraduate students in exam situations: the role of gender and musical instrument. Procedia-Social and Behavioral Sciences, 33, 448-452. https://doi.org/10.1016/j.sbspro.2012.01.161

James, I. (1998). Western orchestral musicians are highly stressed. Resonance: International Music Council, 26, 19-20.

Johnson, R. B., y Onwuegbuzie, A. J. (2004). Mixed methods research: A research paradigm whose time has come. Educational researcher, 33(7), 14-26. https://doi.org/10.3102/0013189X033007014

Kaleńska-Rodzaj, J. (2019). Pre-performance emotions and music performance anxiety beliefs in young musicians. Research Studies in Music Education. https://doi.org/10.1177/1321103X19830098

Kenny, D. T. (2005). A Systematic Review of Treatments for Music Performance Anxiety. Anxiety, Stress, and Coping, 18(3), 183-208. https://doi.org/10.1080/10615800500167258

Kenny, D. T. (2009). The factor structure of the revised Kenny Music Performance Anxiety Inventory. Presentado en International Symposium on Performance Science; Auckland, Nueva Zelanda.

Kenny, D. T. (2011). The Psychology of Music Performance Anxiety. Oxford, Reino Unido: Oxford University Press. https://doi. org/10.1093/acprof:oso/9780199586141.001.0001

Kenny, D. T. (2016). Music Performance Anxiety: Theory, Assessment and Treatment. Saarbrücken, Alemania: LAP Lambert Academic Publishing.

Kenny, D., Driscoll, T., y Ackermann, B. (2014). Psychological well-being in professional orchestral musicians in Australia: A descriptive population study. Psychology of Music, 42(2), 210-232. https://doi.org/10.1177/0305735612463950 
Kenny, D. T., y Osborne, M. S. (2006). Music performance anxiety: New insights from young musicians. Advances in cognitive psychology, 2(2-3), 103-112. https://doi.org/10.2478/v10053-008-0049-5

Kobori, O., Yoshie, M., Kudo, K., y Ohtsuki, T. (2011). Traits and cognitions of perfectionism and their relation with coping style, effort, achievement, and performance anxiety in Japanese musicians. Journal of anxiety disorders, 25(5), 674-679. https://doi. org/10.1016/j.janxdis.2011.03.001

Koeppen, A. S. (1974). Relaxation training for children. Elementary School Guidance \& Counseling, 9(1), 14-21.

McMillan, J.H., y Schumacher, S. (2011). Investigación educativa: una introducción conceptual (5a Ed.). Madrid, España: Pearson Educación.

Nagel, J. J. (2010). Treatment of music performance anxiety via psychological approaches: A review of selected CBT and psychodynamic literature. Medical Problems of Performing Artists, 25(4), 141-148.

Navarro, F. (2018). 126 dinámicas de educación emocional. Málaga: La botica del orientador. Recuperado de http://orientafer. blogspot.com/2011/09/126-dinamicas-de-educacion-emocional.html

Orejudo, S., Zarza-Alzugaray, F. J., y Casanova, O. (2018). Music performance anxiety. Substance use and career abandonment in Spanish music students. International Journal of Music Education, 36(3), 460-472. https://doi.org/10.1177/0255761418763903

Orejudo, S., Zarza-Alzugaray, F. J., Casanova, O., Rodríguez-Ledo, C., y Mazas, B. (2017). The relation of music performance anxiety (MPA) to optimism, self-efficacy, and sensitivity to reward and punishment: Testing Barlow's theory of personal vulnerability on a sample of Spanish music students. Psychology of Music, 45(4), 570-583. https://doi.org/10.1177/0305735616674791

Organización Mundial de la Salud (2011). Intervención breve vinculada a ASSIST para el consumo problemático de sustancias - Manual para uso en la atención primaria. Washington, D.C., Estados Unidos: Organización Panamericana de la Salud.

Osborne, M. S., y Kenny, D. T. (2008). The role of sensitizing experiences in music performance anxiety in adolescent musicians. Psychology of music, 36(4), 447-462. https://doi.org/10.1177/0305735607086051

Ortiz B. A. (2011a). Music performance anxiety -part 1. A review of its epidemiology. Medical Problems of Performing Artists, 26(2), 102-105.

Ortiz B. A. (2011b). Music performance anxiety-part 2. A review of treatment options. Medical problems of Performing Artists, 26(3), 164-171.

Payne, R. A. (1996). Técnicas de Relajación: Guía Práctica. Barcelona, España: Paidotribo.

Papageorgi, I., Hallam, S. y Welch, G. F. (2007). A conceptual framework for understanding musical performance anxiety. Research studies in music education, 28(1), 83-107. https://doi.org/10.1177/1321103X070280010207

Papageorgi, I., y Kopiez, R. (2012). Psychological and physiological aspects of learning to perform. En The Oxford Handbook of Music Education, Volume 1. Nueva York, EEUU: Oxford University Press. https://doi.org/10.1093/oxford$\mathrm{hb} / 9780199730810.001 .0001$

Rivera S. F. (2017). Convivencia del nivel de significación y tamaño del efecto y otros retos de la práctica basada en la evidencia. Boletín Psicoevidencias, 48.

Ryan, C. (2004). Gender differences in children's experience of musical performance anxiety. Psychology of Music, 32(1), 89103. https://doi.org/10.1177/0305735604039284

Ryan, C. (2005). Experience of musical performance anxiety in elementary school children. International Journal of Stress Management, 12(4), 331. https://doi.org/10.1037/1072-5245.12.4.331

Steptoe, A. (2001). Negative emotions in music making: The problem of performance anxiety. En P. N. Juslin y J. A. Sloboda (Eds.), Music and emotion: Theory and research (pp. 291-307). Oxford, Reino Unido: Oxford University Press.

Urruzola, M. V., y Bernaras, E. (2020). Aprendizaje musical y ansiedad escénica en edades tempranas: 8-12 años. Revista de Psicodidáctica, 25(1), 76-83. https://doi.org/10.1016/j.psicod.2019.10.001

Viejo, C. y Laucirica, A. (2016). Entrenamiento mental, relajación e intervención educativa para la reducción del miedo escénico en estudiantes de flauta travesera. Revista Electrónica de LEEME, 37, 63-80. http://musica.rediris.es/leeme.

Wiedemann, A., Vogel, D., Voss, C., y Hoyer, J. (2021). How does music performance anxiety relate to other anxiety disorders? Psychology of Music. https://doi.org/10.1177/0305735620988600

Yeager, D. S., Romero, C., Paunesku, D., Hulleman, C. S., Schneider, B., Hinojosa, C., ... y Trott, J. (2016). Using design thinking to improve psychological interventions: The case of the growth mindset during the transition to high school. Journal of educational psychology, 108(3), 374. https://doi.org/10.1037/edu0000098

Yoshie, M., Kudo, K., y Ohtsuki, T. (2008). Effects of psychological stress on state anxiety, electromyographic activity, and arpeggio performance in pianists. Medical Problems of Performing Artists, 23, 120-132. https://doi.org/10.1037/edu0000098

Yoshie, M., Kudo, K., Murakoshi, T., y Ohtsuki, T. (2009). Music performance anxiety in skilled pianists: Efects of social-evaluative performance situation on subjective, autonomic, and electromyographic reactions. Experimental Brain Research, 199(2), 117- 126. https://doi.org/10.21091/mppa.2008.3024

Zarza-Alzugaray, F. J., Casanova, L. O., y Orejudo, H. S. (2016a). Estudios de música en los conservatorios superiores y ansiedad escénica en España. Revista Electrónica Complutense De Investigación En Educación Musical - RECIEM, 13, 50-63. https:// doi.org/10.5209/RECIEM.49442

Zarza-Alzugaray, F. J., Casanova, L. O., y Orejudo, H. S. (2016b). Music performance anxiety and related psychological constructs. Students of five spanish music conservatories. Revista Internacional De Educación Musical, 4(1), 13-24. https://doi. org/10.12967/RIEM-2016-4-p013-024

Zarza-Alzugaray, F.J., Casanova-López, O., y Robles-Rubio, J.E. (2016). Relación entre ansiedad escénica, perfeccionismo y calificaciones en estudiantes del Título Superior de Música. ReiDoCrea, 5, 16-21. https://doi.org/10.30827/Digibug.39735 
Zarza-Alzugaray, F. J., Orejudo, H. S., Casanova, L. O., y Aparicio-Moreno, L. (2017). Music Performance Anxiety in adolescence and early adulthood: Its relation with the age of onset in musical training. Psychology of Music, 46(1), 18-32. https:// doi.org/10.1177/0305735617691592

Zarza-Alzugaray, F. J., Orejudo, H. S., Casanova, L. O., y Mazas, G. B. (2015). Kenny Music Performance anxiety inventory: Confirmatory factor analysis of the Spanish version. Psychology of Music, 44(3), 340-352. https://doi.org/10.1177/0305735614567932 